\title{
Imaging Tumor Burden in the Brain with ${ }^{89} \mathrm{Zr}$-Transferrin
}

\author{
Michael J. Evans ${ }^{1}$, Jason P. Holland ${ }^{2}$, Samuel L. Rice ${ }^{2}$, Michael G. Doran ${ }^{2}$, Sarah M. Cheal ${ }^{2}$, Carl Campos ${ }^{1}$, \\ Sean D. Carlin ${ }^{2}$, Ingo K. Mellinghoff ${ }^{1}$, Charles L. Sawyers ${ }^{1,3}$, and Jason S. Lewis ${ }^{2,4,5}$ \\ ${ }^{1}$ Human Oncology and Pathogenesis Program, Memorial Sloan-Kettering Cancer Center, New York, New York; ${ }^{2}$ Department of \\ Radiology, Memorial Sloan-Kettering Cancer Center, New York, New York; ${ }^{3}$ Howard Hughes Medical Institute, Memorial \\ Sloan-Kettering Cancer Center, New York, New York; ${ }^{4}$ Program in Molecular Pharmacology and Chemistry, Memorial Sloan- \\ Kettering Cancer Center, New York, New York; and ${ }^{5}$ Radiochemistry and Imaging Sciences Service, Memorial Sloan-Kettering \\ Cancer Center, New York, New York
}

\begin{abstract}
A noninvasive technology that indiscriminately detects tumor tissue in the brain could substantially enhance the management of primary or metastatic brain tumors. Although the documented molecular heterogeneity of diseases that initiate or eventually deposit in the brain may preclude identifying a single smoking-gun molecular biomarker, many classes of brain tumors are generally avid for transferrin. Therefore, we reasoned that applying a radiolabeled derivative of transferrin $\left({ }^{89} \mathrm{Zr}\right.$-labeled transferrin) may be an effective strategy to more thoroughly identify tumor tissue in the brain, regardless of the tumor's genetic background. Methods: Transferrin was radiolabeled with ${ }^{89} \mathrm{Zr}$, and its properties with respect to human models of glioblastoma multiforme were studied in vivo. Results: In this report, we show proof of concept that ${ }^{89} \mathrm{Zr}$-labeled transferrin $\left({ }^{89} \mathrm{Zr}\right.$-transferrin) localizes to genetically diverse models of glioblastoma multiforme in vivo. Moreover, we demonstrate that ${ }^{89} \mathrm{Zr}$-transferrin can detect an orthotopic lesion with exceptional contrast. Finally, the tumor-to-brain contrast conferred by ${ }^{89} \mathrm{Zr}$-transferrin vastly exceeded that observed with ${ }^{18} \mathrm{~F}-\mathrm{FDG}$, currently the most widely used radiotracer to assess tumor burden in the brain. Conclusion: The results from this study suggest that ${ }^{89} \mathrm{Zr}$-transferrin could be a broadly applicable tool for identifying and monitoring tumors in the brain, with realistic potential for near-term clinical translation.
\end{abstract}

Key Words: ${ }^{89} \mathrm{Zr}$; transferrin; positron emission tomography; glioma; molecular imaging

J Nucl Med 2013; 54:90-95

DOI: 10.2967/jnumed.112.109777

$\mathbf{T}$ he effective management of tumors in the brain imposes several unique clinical challenges. For example, the complete surgical resection of a primary brain tumor-a critical factor influencing prognosis-is often complicated by atypical tumor margins or proximity to essential neurologic

\footnotetext{
Received Jun. 5, 2012; revision accepted Aug. 9, 2012.

For correspondence or reprints contact: Jason S. Lewis, Radiochemistry and Imaging Sciences Service, Department of Radiology, Memorial SloanKettering Cancer Center, 1275 York Ave., New York, NY 10065.

E-mail: lewisj2@mskcc.org

Published online Dec. 12, 2012.

COPYRIGHT @ 2013 by the Society of Nuclear Medicine and Molecular Imaging, Inc.
}

tissue $(1,2)$. Moreover, because the blood-brain barrier can obstruct the delivery of systemically administered therapeutics, the pharmacokinetic limitations of drugs designed to address primary or metastatic brain tumors can severely dampen clinical responses (3).

With some success, noninvasive imaging modalities have been invoked to address these challenges. One of the most visible examples of progress has been the use of MR imaging to localize a tumor mass or to monitor tumor burden after therapy (4). However, the low sensitivity of this modality, and its inability to distinguish tumors from nonmalignant pathologies (infection), precludes its application to some common clinical issues (e.g., detection of residual or recurrent subclinical disease).

Because of its comparatively lower detection limit, and its ability to intercalate tumor biology independent of morphologic changes, nuclear medicine technologies (e.g., PET) are regarded as an attractive complement to anatomic imaging. Indeed, several clinical studies have been conducted with common $\left({ }^{18} \mathrm{~F}-\mathrm{FDG}\right)$ and investigational radiotracers $\left(3^{\prime}-\right.$ deoxy- $3^{\prime}-{ }^{18} \mathrm{~F}$-fluorothymidine, ${ }^{11} \mathrm{C}$-labeled amino acids), resulting in some degree of disease contrast and effectively establishing proof of concept (5). However, shortcomings have been documented and are generally attributed to obfuscating uptake in normal brain tissue, ongoing questions concerning mechanism of action, and the practical limitations of studying radiotracers with rapid decay kinetics.

On the basis of these observations, we hypothesized that ${ }^{89} \mathrm{Zr}$-transferrin, a radiotracer we previously developed to target transferrin receptor (TFRC) in prostate cancer (6), could be a generally useful tool for the detection and monitoring of tumor burden in the brain. Indeed, there is a known tropism of transferrin for primary and metastatic brain tumors $(7,8)$, leading several groups to exploit this property therapeutically by coupling transferrin to anticancer drugs to foster tumor-specific delivery (9-12). Moreover, our previous work with this radiotracer (and with ${ }^{89} \mathrm{Zr}$-labeled monoclonal antibodies) resulted in high-contrast images of tumors, with generally low uptake in normal tissues $(13,14)$. Part of the success can be attributed to ${ }^{89} \mathrm{Zr}$ itself-the radionuclide has highly attractive physical properties such as its long half- 
life $(\sim 78 \mathrm{~h})$, which is well suited to the length of time required for large biomolecules such as transferrin to distribute in vivo (15). Collectively, these observations led us to profile the avidity of primary brain tumor models for ${ }^{89} \mathrm{Zr}$-transferrin.

\section{MATERIALS AND METHODS}

\section{Reagents, Cells, and Mice}

All chemicals, unless otherwise stated, were purchased from Sigma Aldrich and used without further purification. Water (Millipore) was purified with a $10-\mathrm{cm}$ column of Chelex resin (Bio-Rad). TS543 was obtained from Dr. Cameron Brennan of Memorial Sloan-Kettering Cancer Center (MSKCC) and cultured in a NeuroCult NS-A Proliferation Kit (human) from Stemcell Technologies, Inc. U87 MG, SF268, and LN-18 were purchased (American Type Culture Collection) and cultured according to manufacturer's recommendations. All cell lines were authenticated for TFRC expression by immunoblot and reverse transcriptase polymerase chain reaction (Supplemental Fig. 1; supplemental materials are available online only at http://jnm.snmjournals. org). Otherwise, no further authentication was performed, because cell lines were studied within 6 mo of their acquisition from the stated sources. Five-week-old male ICR severe-combined immunodeficiency mice were obtained from Taconic Farms (model ICRSC-M). All animal experiments were conducted in compliance with Institutional Animal Care and Use Committee guidelines at MSKCC.

\section{Transferrin Conjugation and Radiolabeling}

Human transferrin (Sigma Aldrich) was conjugated to desferrioxamine $\mathrm{B}$ (Calbiochem) radiolabeled with ${ }^{89} \mathrm{Zr}$ and purified as previously described (6). The radiochemical purity of ${ }^{89} \mathrm{Zr}$-transferrin was consistently greater than $99 \%$, and the specific activity was approximately $300 \mathrm{MBq} / \mathrm{mg}$. Radioactivity was measured with a Capintec CRC-15R dose calibrator.

\section{Animal Studies}

Animals were inoculated with tumors by subcutaneous injection of $1.0 \times 10^{6}$ cells in $200 \mu \mathrm{L}$ of Matrigel (Becton Dickinson) and cell culture medium (1:1 v/v), and studies commenced when tumor volumes were approximately $200 \mathrm{~mm}^{3}$. For intracranial injections, mice were anesthetized using ketamine (100 $\mathrm{mg} / \mathrm{kg}$ ) and xylazine $(10 \mathrm{mg} / \mathrm{kg})$ by intraperitoneal injection. A toe pinch was used to determine reflex response. The head was then shaved and the surgical area cleaned 3 times with alcohol and povidone-iodine. An incision was made along the midline of the skull, and the injection site was drilled into the skull at coordinates $1 \mathrm{~mm}$ posterior from the bregma and $1.5 \mathrm{~mm}$ right of the midline. The mice were then placed in a stereotactic frame. A Hamilton syringe was inserted $3.5 \mathrm{~mm}$ into the brain and was retracted $0.5 \mathrm{~mm}$ to create room for the cells, and $5 \times 10^{4}$ cells (constituted in $2 \mu \mathrm{L}$ ) were injected into the brain. The mice were monitored daily for physical symptoms of tumor growth, and tumor onset was confirmed by MR imaging.

\section{Biodistribution Studies}

The mice were injected intravenously in the tail with ${ }^{89} \mathrm{Zr}$ transferrin or ${ }^{18} \mathrm{~F}-\mathrm{FDG}(1.85 \mathrm{MBq} / \mathrm{mouse} ; 6.2 \mu \mathrm{g}$ of protein) and were kept fasting $4 \mathrm{~h}$ before ${ }^{18} \mathrm{~F}$-FDG injection. They were euthanized by $\mathrm{CO}_{2}$ gas asphyxiation at multiple time points after injection, and tissues (including the tumor) were removed, rinsed in water, and weighed. Activity was then measured with a $\gamma$ counter (Perkin Elmer). Standard syringes of the formulation injected into the animals were used to determine the total number of counts per minute in the dissected tissues. Count data were background- and decay-corrected, and the tissue uptake was measured in units of percentage injected dose per gram (\% ID/g).

\section{Small-Animal Imaging Experiments}

For tumor-bearing animals, ${ }^{89} \mathrm{Zr}$-transferrin or ${ }^{18} \mathrm{~F}-\mathrm{FDG}$ $(\sim 11.1 \mathrm{MBq} /$ mouse) was administered intravenously in 200 $\mu \mathrm{L}$ of sterile saline. PET experiments were conducted at multiple time points after injection on a microPET Focus 120 scanner (Concorde Microsystems). Before images were acquired, the mice were anesthetized with $1 \%-2 \%$ isoflurane (Baxter Healthcare) oxygen gas mixture and placed on the scanner bed. Listmode data were acquired for between 10 and $30 \mathrm{~min}$. Data were sorted into 2-dimensional histograms by Fourier rebinning, and transverse images were reconstructed by filtered backprojection into a $128 \times 128 \times 63(0.72 \times 0.72 \times 1.3 \mathrm{~mm})$ matrix. The image data were normalized to correct for nonuniformity of response of the PET, dead-time count losses, positron branching ratio, and physical decay to the time of injection, but no attenuation, scatter, or partial-volume averaging correction was applied. An empirically determined system calibration factor (in units of [MBq/ $\mathrm{mL}] /[\mathrm{cps} /$ voxel]) for mice was used to convert voxel counting rates to activity concentrations. The resulting image data were then normalized to the administered activity to parameterize images in terms of $\% \mathrm{ID} / \mathrm{g}$. Manually drawn 2-dimensional regions of interest were used to determined the maximum and mean (decaycorrected) $\% \mathrm{ID} / \mathrm{g}$ with the ASIPro software package (Concorde Microsystems).

MR images were acquired on a Bruker 4.7-T Biospec scanner operating at $200 \mathrm{MHz}$ and equipped with a 12-cm gradient coil (400 mT/m ID; Bruker Biospin MRI GmbH). A custom-built quadrature birdcage resonator with an internal diameter of $32 \mathrm{~mm}$ was used for radiofrequency excitation and acquisition (Stark Contrast MRI Coils Research Inc.). Mice were anesthetized with oxygen and $1 \%$ isoflurane gas. Animal breathing was monitored using a small-animal physiologic monitoring system (SA Instruments, Inc.). T2-weighted scout images along 3 orthogonal orientations were first acquired for animal positioning. A T2-weighted fast spin-echo sequence (rapid acquisition with relaxation enhancement [RARE]was used to acquire axial mouse cranial images with a slice thickness of $0.8 \mathrm{~mm}$ and a field of view of $30 \times 34 \mathrm{~mm}$ with a spatial resolution of $117 \times 133 \mu \mathrm{m}$. The following acquisition parameters were used: repetition time, $4.5 \mathrm{~s}$; echo time, $40 \mathrm{~ms}$; RARE factor, 8; and acquisition time, $20 \mathrm{~min}$.

\section{Digital Autoradiography and Histology}

Serial $10-\mu \mathrm{m}$-thick sections were cut at $-20^{\circ} \mathrm{C}$ in an HM 500 $\mathrm{M}$ cryostat (Microm) at 1-mm intervals throughout each tumor and immediately deposited onto a glass plate. Digital autoradiography was performed with a FujiFilm BAS-1800 II device (Fuji Photo Film) for an appropriate exposure time. The image reader creates 16-bit gray-scale digital images with 50 - $\mu$ m pixel resolution. These images are then converted to tagged image format files for subsequent registration.

After digital autoradiography exposure, sections were subsequently stained with hematoxylin and eosin and visualized with 


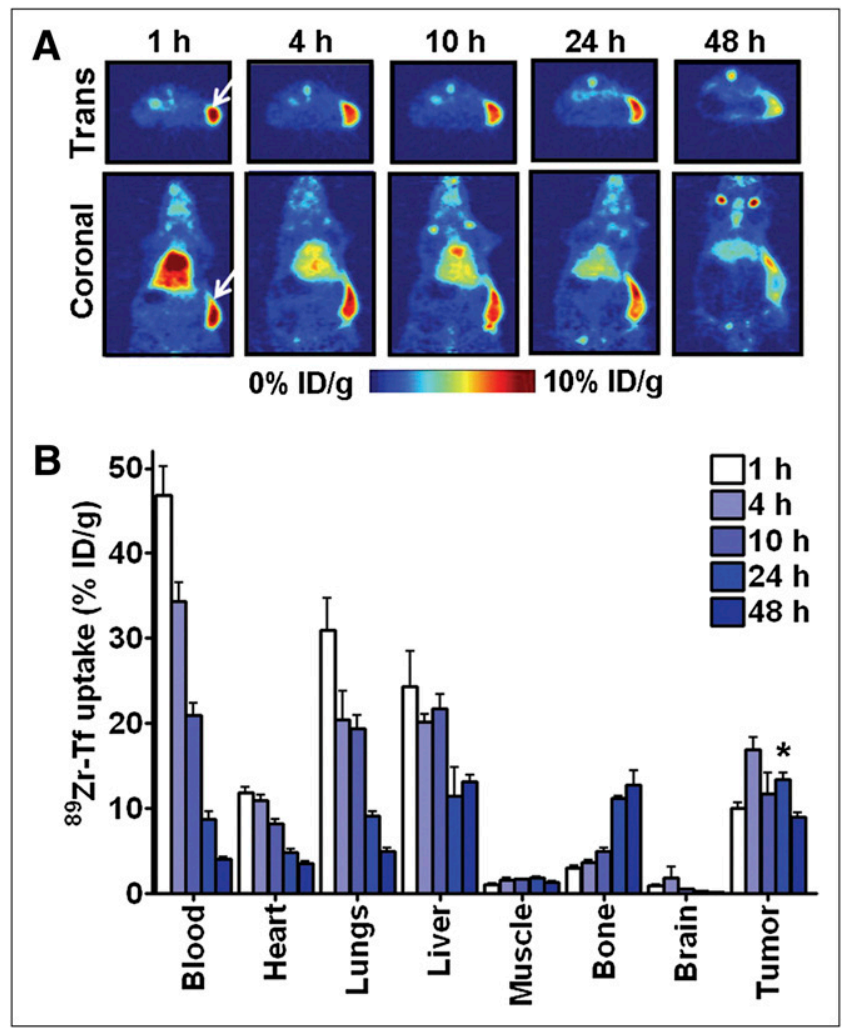

FIGURE 1. Temporal analysis of ${ }^{89} \mathrm{Zr}$-transferrin in tumor-bearing mice. (A) Representative PET images of tumor-bearing mouse show high and persistent uptake of ${ }^{89} \mathrm{Zr}$-transferrin in subcutaneous TS543 xenografts. White arrows indicate position of tumor (T) or liver (L). (B) Biodistribution data ( $n=5$ per time point) from selected panel of tissues show high uptake of ${ }^{89} \mathrm{Zr}$-transferrin in tumor, compared with other tissues. Although ${ }^{89} \mathrm{Zr}$-transferrin continuously depletes from blood-rich tissues over time (heart, lungs), high and persistent uptake of ${ }^{89} \mathrm{Zr}$-transferrin is observed in tumor over $48 \mathrm{~h}$. Note also low uptake of ${ }^{89} \mathrm{Zr}$-transferrin in normal murine brain. ${ }^{*} P<$ 0.01 , compared with biodistribution data in blood. $\mathrm{Tf}=$ transferrin; Trans $=$ transverse slice.

a BX 60 microscope (Olympus America) equipped with a $10 \times / 0.30$ UPlanF objective, a ProScan II automated stage (Prior Scientific Instruments), and a CC12 Soft Imaging Systems camera (Olympus America). Microsuite Five software (Olympus America) was used to align the individual image frames and render these into a single montage of the entire tumor section. Histologic and digital autoradiography images obtained from the same section were then registered using Photoshop software (version 7.0; Adobe).

\section{Statistical Analysis}

Data were analyzed using the unpaired, 2-tailed Student $t$ test. Differences at the 95\% confidence level $(P<0.05)$ were considered to be statistically significant.

\section{RESULTS}

\section{In Vivo Studies with Subcutaneous Glioblastoma Multiforme (GBM) Model}

Because elevated transferrin uptake is regarded to be a common feature of primary brain tumors, we first asked if ${ }^{89} \mathrm{Zr}$-transferrin can localize to a subcutaneous model of



FIGURE 2. ${ }^{89} \mathrm{Zr}$-transferrin detects orthotopic model of GBM in vivo. (A) Representative MR slices showing tumor development in murine brain. Fourteen days after inoculation, tumor development was confirmed visually with MR imaging, before development of clinical symptoms in cohort. Shown are 2 consecutive coronal slices from 1 animal. Region of contrast showing tumor mass is indicated with red arrow. All MR data are available on request. (B) Representative PET images of tumor-bearing mouse show high contrast achieved with ${ }^{89} \mathrm{Zr}$-transferrin in TS543 graft inoculated in right hemisphere of murine brain. Images were acquired at $24 \mathrm{~h}$ after injection of ${ }^{89} \mathrm{Zr}$-transferrin. White arrows indicate position of tumor (T) or liver (L). (C) Histology (left) and autoradiography (right) from murine brain bearing TS543 tumor in right hemisphere. Brain is shown at $\times 4$ magnification, and intensity of radiotracer localization is depicted using semiquantitative red (high) to blue (low) scale. (D) Biodistribution data of right (tumor-bearing) and left (normal) brain hemispheres from cohort of mice $(n=5)$ treated with ${ }^{89} \mathrm{Zr}$-transferrin. After euthanasia, brain was bisected along medial longitudinal fissure, and ${ }^{89} \mathrm{Zr}$-transferrin uptake in each piece was counted separately on $\gamma$-counter. Data were acquired from animals euthanized at $24 \mathrm{~h}$ after injection of ${ }^{89} \mathrm{Zr}$-transferrin. ${ }^{\star} P<0.05$, compared with normal (left) hemispheres. $\mathrm{Tf}=$ transferrin; Trans $=$ transverse slice.

human GBM in vivo. The mice were inoculated with subcutaneous xenografts of TS543. After tumor formation, the animals were treated with ${ }^{89} \mathrm{Zr}$-transferrin via tail vein injection, and PET and biodistribution studies were conducted at multiple time points after injection. Region-of-interest analysis of temporal PET studies showed early and persistent uptake, with peak intratumoral uptake observed at $4 \mathrm{~h}$ (Fig. 1A; Supplemental Fig. 1; Supplemental Table 1). Biodistribution studies at multiple time points corroborated the PET data, with intratumoral uptake of ${ }^{89} \mathrm{Zr}$-transferrin exceeding blood-pool activity after $24 \mathrm{~h}$, and a maximum tumor-tomuscle ratio of approximately 7:1 was observed (Fig. 1B; Supplemental Fig. 2; Supplemental Table 2). Notably, little 
uptake of ${ }^{89} \mathrm{Zr}$-transferrin was detected in the brain, and the tumor-to-brain ratio at $24 \mathrm{~h}$ after injection was approximately 50:1 (Supplemental Table 2). Collectively, these data highlight the avidity of ${ }^{89} \mathrm{Zr}$-transferrin for a preclinical model of GBM.

\section{In Vivo Studies with Orthotopic GBM Model}

On the basis the biodistribution data, we next asked if systemically administered ${ }^{89} \mathrm{Zr}$-transferrin could detect an orthotopic brain tumor. Mice were inoculated with TS543 via an intracranial injection into the right hemisphere. After $14 \mathrm{~d}$, the topography of the lesion was documented by MR imaging (Fig. 2A), and the animals were subsequently injected via the tail vein with ${ }^{89} \mathrm{Zr}$ transferrin. The orthotopic tumor was visualized with high contrast by PET at $24 \mathrm{~h}$ after radiotracer injection (Fig. 2B; Supplemental Table 3). In comparison, no compelling contrast was observed in the brains of animals receiving a sham injection, despite their also having a mechanically compromised blood-brain barrier (Fig. 2B).

Ex vivo analysis of brain tissue supported the wholebody imaging results. For instance, autoradiography showed a compelling overlap between tumor tissue (defined by hematoxylin and eosin) and radiotracer accumulation, with no obvious radioactivity associated with normal brain (Fig. 2C; Supplemental Fig. 3). In addition, PET imaging of the brain ex vivo after bisection along the medial longitudinal fissure showed a higher signal associated with the right (tumor-bearing) versus left (normal) hemisphere (Supplemental Fig. 4). Finally, biodistribution studies from the entire cohort of mice $(n=4)$ confirmed higher radioactivity in the right hemisphere (Fig. 2D; Supplemental Table 3).

\section{Survey of Subcutaneous GBM Models with ${ }^{89} \mathrm{Zr}$-Transferrin and ${ }^{18} \mathrm{~F}-\mathrm{FDG}$}

The previous studies were conducted using a single GBM model. To determine whether ${ }^{89} \mathrm{Zr}$-transferrin uptake in tumors can be documented more broadly, we profiled the avidity of several models of GBM that we had annotated for TFRC status for ${ }^{89} \mathrm{Zr}$-transferrin (Fig. 3A). These models were chosen because they partially recapitulate the molecular diversity displayed in clinical disease. Subcutaneous xenografts of U87 MG, LN-18, and SF268 were established in mice, and at $24 \mathrm{~h}$ after injection, uptake of ${ }^{89} \mathrm{Zr}$-transferrin was assessed by biodistribution (Fig. 3B). At $24 \mathrm{~h}$, all models showed evidence of radiotracer uptake, albeit variable among the panel. One shared feature was the high tumor-to-brain ratios conferred by this radiotracer, in all cases exceeding approximately 15:1 (Fig. 3C). This property distinguished ${ }^{89} \mathrm{Zr}$-transferrin from ${ }^{18} \mathrm{~F}-\mathrm{FDG}$, because although all GBM models were also avid for ${ }^{18}$ F-FDG (Figs. 3B and 3D; Supplemental Table 4), the tumor-to-brain ratio was comparatively poorer $(\sim 1: 1)$. Collectively, these results underscore a potential clinical utility for invoking ${ }^{89} \mathrm{Zr}$-transferrin PET to document tumor burden.

\section{DISCUSSION}

In this study, we obtained proof-of-concept data from preclinical GBM models advancing ${ }^{89} \mathrm{Zr}$-transferrin as a credible imaging tool to assess tumor burden in the brain. In our work with ${ }^{89} \mathrm{Zr}$-transferrin and other radiotracers, we have learned that a deep appreciation of the pathobiology underlying tumor avidity for the radiotracer can significantly expand its application beyond detecting the presence or absence of disease (6,16-18). Consequently, one frontier in the future development of ${ }^{89} \mathrm{Zr}$-transferrin

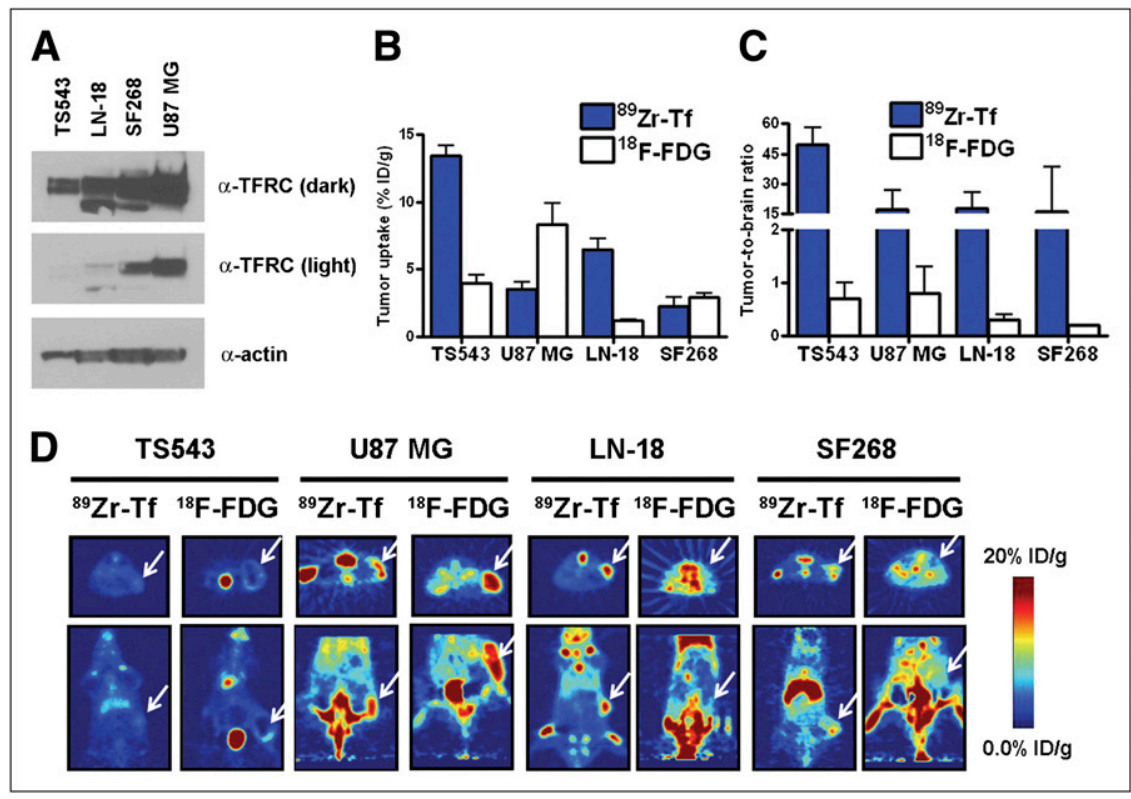

FIGURE 3. ${ }^{89} \mathrm{Zr}$-transferrin detects multiple subcutaneous GBM models with exceptional tumor-to-brain ratios in vivo. (A) Western blot showing relative expression of TFRC in 4 glioblastoma models used in this study. (B) Biodistribution data from animals used in PET study $(n=5$ per group). Tissues were acquired immediately after PET scan. (C) Tumor-to-brain ratios calculated from biodistribution data show high degree of contrast conferred by ${ }^{89} \mathrm{Zr}$-transferrin in all models. As expected, tumor-to-brain ratio for ${ }^{18} \mathrm{~F}$ FDG was poor. (D) Representative PET images of mice bearing subcutaneous TS543, U87 MG, LN-18, or SF268 xenografts. Separate treatment arms received ${ }^{89} \mathrm{Zr}$-transferrin or ${ }^{18} \mathrm{~F}-\mathrm{FDG}$ and were imaged at 24 or $1 \mathrm{~h}$ after injection, respectively. Position of tumor is indicated with arrow. $\mathrm{Tf}=$ transferrin. 
is to identify the molecular determinants governing its seemingly broad (and variable) affinity for brain tumors. Because it has recently become clear that overexpression of TFRC or elevated transferrin uptake can be immediately linked to the pathologic activation of myelocytomatosis cellular oncogene (MYC) or phosphoinositide-3-kinase (PI3K) pathway signaling $(19,20)$, one compelling hypothesis in the case of GBM is that PTEN loss (an event occurring in $\sim 70 \%$ of patients with GBM (21)) may drive ${ }^{89} \mathrm{Zr}$-transferrin uptake. Further work is ongoing to systematically address this hypothesis; however, it is interesting to note that basal ${ }^{89} \mathrm{Zr}$-transferrin uptake was found to be higher in LN-18 tumors (PTEN wild type) than in U87 MG tumors (PTEN null), suggesting that other molecular events may influence transferrin uptake. Regardless, defining the molecular basis of ${ }^{89} \mathrm{Zr}$-transferrin avidity will almost certainly strengthen the rationale for its translation.

Although ${ }^{67} \mathrm{Ga}$-citrate (a radiotracer that binds transferrin in serum to target tumors (22)) has been applied effectively to detect spontaneous lymphoma metastases in the central nervous system (23), to our knowledge this is the first report showing that transferrin can be leveraged to detect primary brain tumors. Our findings are particularly germane to the neurooncology community, because the frustratingly poor performance of targeted therapies in treating primary brain tumors has sustained interest in alternative therapeutic strategies (24). Among these, transferrin-CRM107 (human transferrin conjugated to a mutant diphtheria toxin) was well tolerated in patients with glioma at doses sufficient to confer clear tumor regressions by MR imaging (25). Although a phase III clinical trial was recently suspended as evidence mounted suggesting transferrin-CRM107 would not be an improvement over the standard of care, several highly potent secondgeneration transferrin drug conjugates are undergoing early clinical development (9-12). In this regard (and in addition to our originally stipulated rationale for its development), a radiotracer such as ${ }^{89} \mathrm{Zr}$-transferrin may be a powerful pharmacodynamic marker to understand dosing in future clinical trials and, more speculatively, could behave as a predictive biomarker of response on the basis of the pretherapy radiotracer retention in a tumor. Indeed, the fact that no obvious correlation between TFRC expression and ${ }^{89} \mathrm{Zr}$-transferrin uptake in the tumor was observed in the small panel of models that we surveyed strongly suggests that imaging may be necessary to identify patients most likely to benefit from a transferrin-drug conjugate.

\section{CONCLUSION}

In this study, we present proof-of-concept data showing that ${ }^{89} \mathrm{Zr}$-transferrin localized to multiple GBM models, regardless of genetic background. Notably, systemically administered ${ }^{89} \mathrm{Zr}$-transferrin was sufficient to detect an orthotopic lesion in murine brain, suggesting that ${ }^{89} \mathrm{Zr}$ - transferrin PET may be a practical assay, particularly in the context of a compromised blood-brain barrier. Finally, tumor-to-brain contrast ratios were exceptional and vastly outperformed ${ }^{18} \mathrm{~F}-\mathrm{FDG}$ in all models.

\section{DISCLOSURE}

The costs of publication of this article were defrayed in part by the payment of page charges. Therefore, and solely to indicate this fact, this article is hereby marked "advertisement" in accordance with 18 USC section 1734. This study was supported in part by the Brain Tumor Center of MSKCC, the Geoffrey Beene Cancer Research Center of MSKCC, a training grant from the National Institutes of Health (R25-CA096945), the U.S. Department of Energy (DE-SC0002456), the Howard Hughes Medical Institute, the National Brain Tumor Society, and an Advanced Clinical Research Award from the American Society of Clinical Oncology. Technical services provided by the MSKCC SmallAnimal Imaging Core Facility were supported by the National Institutes of Health (R24-CA83084, P30-CA08748, and P50CA92629). No other potential conflict of interest relevant to this article was reported.

\section{ACKNOWLEDGMENTS}

We thank Drs. Igor Vivanco, Barbara Oldrini, NagaVaraKishore Pillarsetty, and Pat Zanzonico for informative discussions and Valerie Longo for technical assistance. We thank Dr. Carl Le and Dov Winkleman for recording of the MRI data. Finally, we thank Dr. Cameron Brennan and Alicia Pedraza for establishing and distributing the TS543 cell line.

\section{REFERENCES}

1. Bucci MK, Maity A, Janss AJ, et al. Near complete surgical resection predicts a favorable outcome in pediatric patients with nonbrainstem, malignant gliomas: results from a single center in the magnetic resonance imaging era. Cancer. 2004; 101:817-824.

2. Stupp R, Hegi ME, van den Bent MJ, et al. Changing paradigms: an update on the multidisciplinary management of malignant glioma. Oncologist. 2006;11: $165-180$.

3. Denora N, Trapani A, Laquintana V, Lopedota A, Trapani G. Recent advances in medicinal chemistry and pharmaceutical technology: strategies for drug delivery to the brain. Curr Top Med Chem. 2009;9:182-196.

4. Waldman AD, Jackson A, Price SJ, et al. Quantitative imaging biomarkers in neuro-oncology. Nat Rev Clin Oncol. 2009;6:445-454.

5. la Fougère C, Suchorska B, Bartenstein P, Kreth FW, Tonn JC. Molecular imaging of gliomas with PET: opportunities and limitations. Neuro-oncol. 2011; 13:806-819.

6. Holland J, Evans MJ, Rice SL, Wongvipat J, Sawyers CL, Lewis JS. Annotating MYC oncogene status with ${ }^{89} \mathrm{Zr}$-transferrin. Nat Med. 2012;18:15861591.

7. Calzolari A, Oliviero I, Deaglio S, et al. Transferrin receptor 2 is frequently expressed in human cancer cell lines. Blood Cells Mol Dis. 2007;39:82-91.

8. Recht L, Torres CO, Smith TW, Raso V, Griffin TW. Transferrin receptor in normal and neoplastic brain tissue: implications for brain-tumor immunotherapy. J Neurosurg. 1990;72:941-945.

9. Eavarone DA, Yu X, Bellamkonda RV. Targeted drug delivery to C6 glioma by transferrin-coupled liposomes. J Biomed Mater Res. 2000;51:10-14.

10. Weaver M, Laske DW. Transferrin receptor ligand-targeted toxin conjugate (TfCRM107) for therapy of malignant gliomas. J Neurooncol. 2003;65:3-13. 
11. Cimini A, Mei S, Benedetti E, et al. Distinct cellular responses induced by saporin and a transferrin-saporin conjugate in two different human glioblastoma cell lines. J Cell Physiol. 2012;227:939-951.

12. Yoon DJ, Kwan BH, Chao FC, et al. Intratumoral therapy of glioblastoma multiforme using genetically engineered transferrin for drug delivery. Cancer Res. 2010;70:4520-4527.

13. Holland JP, Divilov V, Bander NH, Smith-Jones PM, Larson SM, Lewis JS. ${ }^{89} \mathrm{Zr}$ DFO-J591 for immunoPET of prostate-specific membrane antigen expression in vivo. J Nucl Med. 2010;51:1293-1300.

14. Ruggiero A, Holland JP, Hudolin T, et al. Targeting the internal epitope of prostate-specific membrane antigen with ${ }^{89} \mathrm{Zr}-7 \mathrm{E} 11$ immuno-PET. J Nucl Med. 2011;52:1608-1615.

15. Holland JP, Sheh Y, Lewis JS. Standardized methods for the production of high specific-activity zirconium-89. Nucl Med Biol. 2009;36:729-739.

16. Holland JP, Caldas-Lopes E, Divilov V, et al. Measuring the pharmacodynamic effects of a novel Hsp90 inhibitor on HER2/neu expression in mice using $\mathrm{Zr}$ DFO-trastuzumab. PLOS ONE. 2010;5:e8859.

17. Ulmert D, Evans MJ, Holland JP, et al. Imaging androgen receptor signaling with a radiotracer targeting free prostate-specific antigen. Cancer Discov. 2012;2:320-327.

18. Evans MJ, Smith-Jones PM, Wongvipat J, et al. Noninvasive measurement of androgen receptor signaling with a positron-emitting radiopharmaceutical that targets prostate-specific membrane antigen. Proc Natl Acad Sci USA. 2011; 108:9578-9582.

19. Galvez T, Teruel MN, Heo WD, et al. siRNA screen of the human signaling proteome identifies the PtdIns(3,4,5)P3-mTOR signaling pathway as a primary regulator of transferrin uptake. Genome Biol. 2007;8:R142-R153.

20. O'Donnell KA, Yu D, Zeller KI, et al. Activation of transferrin receptor 1 by cMyc enhances cellular proliferation and tumorigenesis. Mol Cell Biol. 2006;26: 2373-2386.

21. Koul D. PTEN signaling pathways in glioblastoma. Cancer Biol Ther. 2008; 7:1321-1325.

22. Larson SM. Mechanisms of localization of gallium-67 in tumors. Semin Nucl Med. 1978;8:193-203.

23. Kosuda S, Kusano S, Ishihara S, et al. Combined ${ }^{201} \mathrm{Tl}$ and ${ }^{67} \mathrm{Ga}$ brain SPECT in patients with suspected central nervous system lymphoma or germinoma: clinical and economic value. Ann Nucl Med. 2003;17:359-367.

24. Mellinghoff IK, Schultz N, Mischel PS, Cloughesy TF. Will kinase inhibitors make it as glioblastoma drugs? Curr Top Microbiol Immunol. October 21, 2011 [Epub ahead of print].

25. Laske DW, Youle RJ, Oldfield EH. Tumor regression with regional distribution of the targeted toxin TF-CRM107 in patients with malignant brain tumors. Nat Med. 1997;3:1362-1368. 